\title{
Determination of reduced Young's modulus of thin films using indentation test
}

Wuzhu YAN, Shifeng WEN, Jun LIU and Zhufeng YUE*

Department of Engineering Mechanics, Northwestern Polytechnical University, Xi'an 710129, China

Manuscript received 27 March 2009; in revised form 18 May 2009

\begin{abstract}
The flat cylindrical indentation tests with different sizes of punch radius were investigated using finite element method (FEM) aimed to reveal the effect of punch size on the indentation behavior of the film/substrate system. Based on the FEM results analysis, two methods was proposed to separate film's reduced Young's modulus from a film/substrate system. The first method was based on a new weight function that quantifies film's and substrate's contributions to the overall mechanical properties of the film/substrate system in the flat cylindrical indentation test. The second method, a numerical approach, including fitting and extrapolation procedures was put forward. Both of the results from the two methods showed a reasonable agreement with the one input FE model. At last, the effect of maximum indentation depth and the surface micro-roughness of the thin film on the reduced Young's modulus of the film/substrate system were discussed. The methods proposed in the present study provide some new conceptions on evaluating other properties of thin films, e.g. creep, for which a flat-ended punch is also employed.

KEY WORDS Indentation test; Reduced Young's modulus; Punch size effect; Weight function; Film/substrate system
\end{abstract}

\section{Introduction}

The indentation test, due to its simplicity, efficiency and non-destruction merits, has been widely used on the determination of mechanical properties of materials. So far, the indentation technique has been used extensively in measuring the Young's modulus, hardness and creep properties of materials ${ }^{[1-3]}$.

With the progressive use of thermal barrier film (TBC) in high temperature alloys, it is essential to develop a simple, fast, and non-destruction method to evaluate the mechanical properties of thin films in a film/substrate-system. Experimental aspect and numerical simulation $^{[4-9]}$ of extracting mechanical properties of the thin films has been discussed in the past three decades.

Yue and $\mathrm{Xu}^{[10-12]}$ studied the indentation behavior of film/substrate system and proposed some numerical approaches to determine mechanical properties of thin films. How-

\footnotetext{
*Corresponding author. Professor, PhD; Tel.: +86 29 88431002; Fax: +86 2988431002.

E-mail address: zfyue@nwpu.edu.cn (Zhufeng YUE)
}

DOI: $10.1016 / \mathrm{S} 1006-7191(08) 60125-8$ 
ever, no explicit relationship between punch size and mechanical parameters of the film/ substrate system has been revealed. So far, the relationship among reduced Young's modulus of the thin film $E_{\mathrm{f}}^{*}$, substrate $E_{\mathrm{s}}^{*}$, film/substrate-system $E_{\mathrm{f} / \mathrm{s}}^{*}$ and the indenter size $R$ has rarely been reported. In this study, a series of finite element analysis of the loadingunloading indentation process was performed for two material systems (single-phase material system and film/substrate-system). For each material system, our FE analysis were carried out for different classes of materials and punch sizes aimed to develop method to separate elastic properties of a film from a film/substrate-system.

\section{Theory Analysis}

It is common practice to extract reduced modulus $E^{*}$ of indented materials from loading-unloading indentation curve, typically an indentation force $v s$ indentation depth curve. Taking into account the Young's modulus of both the tested specimen and the indenter, the reduced Young's modulus can be expressed as

$$
E_{\mathrm{eff}}^{*}=\left(\frac{1-v^{2}}{E}+\frac{1-v_{i}^{2}}{E_{i}}\right)^{-1}
$$

where $E$ and $v$ are the elastic constants of the tested material, $E_{i}$ and $v_{i}$ are the elastic constants of the indenter. In the present study, taking the punch as rigid, the reduced Young's modulus of the tested material can be written as

$$
E^{*}=\left(\frac{1-v^{2}}{E}\right)^{-1}
$$

where $E$ and $v$ are Young's modulus and Poisson's ratio of the tested material respectively. Oliver and Pharr ${ }^{[13]}$ have proposed an equation that relates the reduced Young's modulus $E^{*}$ with the compliance of the unloading curve $m$ immediately before unloading, such as:

$$
E^{*}=\beta \frac{\sqrt{\pi}}{2} \frac{1}{\sqrt{A_{\mathrm{c}}}} \frac{1}{m} ; \quad m=\frac{1}{S}
$$

where $S$ is the indentation stiffness (or initial slope of the unloading curve) shown in Fig.1b, $A_{\mathrm{c}}$ is the projected contact area, $\beta$ is a shape factor, with $\beta=1$ for axisymmetric indenter and $\beta=1.03-1.05$ for indenters with square or rectangular cross-sections.

For the indentation testing, there are a variety of indenter geometrical shapes in use. Considerable researches show that the flat cylindrical punch has more advantages than the spherical punch or sharp punch for the indentation testing of the isotropic materials ${ }^{[14-16,18]}$. The main reason is that the contact area under the punch is constant during the indenting process, while it increases with time for other indenter geometries. In the present study, we take the section of the punch as the projected contact area. From Eq.(3), we have

$$
E^{*}=\frac{\sqrt{\pi}}{2} \frac{S}{\sqrt{\pi R^{2}}}=S /(2 R)=\frac{F_{\max }}{2 R\left(h_{\max }-h_{\mathrm{c}}\right)}
$$

where $h_{\mathrm{c}}$ can be determined by extrapolating the initial slope of the unloading curve to zero load (the corresponding depth is $h_{\mathrm{c}}$, refer to Fig.1b). 

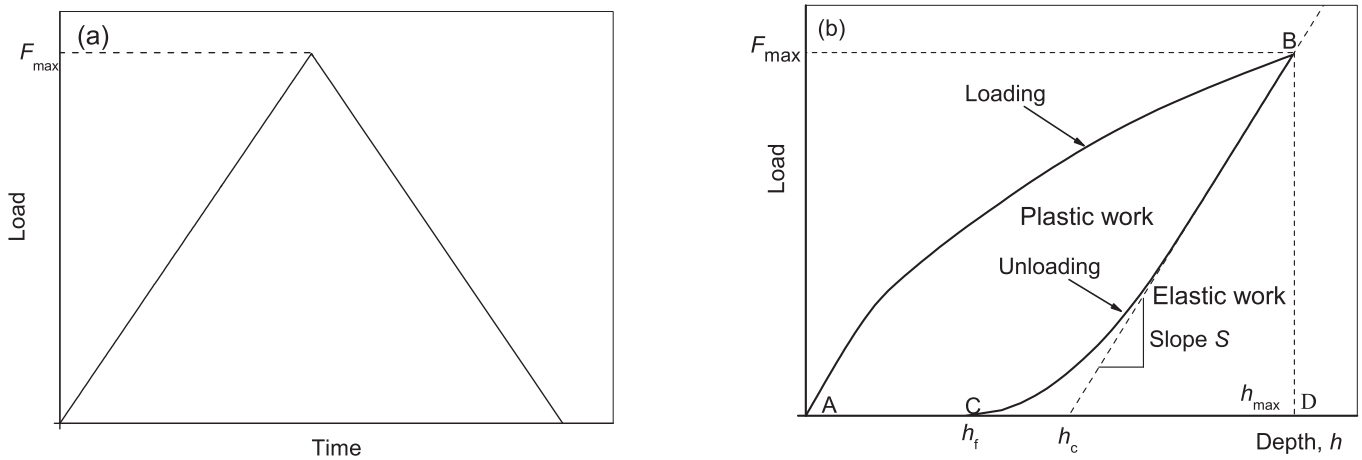

Fig.1 Schematic view of the loading-unloading indentation test: (a) loading history used in the present work; (b) typical loading-unloading curve $F$ - $h$.

The key problem with the characterization of thin films using indentation test is the quantification of the films and the substrate's contributions to the measured properties of film/substrate-system. Weight functions have been used for the derivate of reduced film's Young's modulus. Doerner and Nix ${ }^{[16]}$ proposed an exponential equation:

$$
\left|\frac{1 / E_{\mathrm{f} / \mathrm{s}}^{*}-1 / E_{\mathrm{f}}^{*}}{1 / E_{\mathrm{f}}^{*}-1 / E_{\mathrm{s}}^{*}}\right|=\mathrm{e}^{\left(-\alpha_{1} \frac{T_{\mathrm{f}}}{h}\right)}
$$

where $E_{\mathrm{f}}^{*}, E_{\mathrm{s}}^{*}$ and $E_{\mathrm{f} / \mathrm{s}}^{*}$ are reduced Young's modulus of the film, substrate and film/substratesystem, respectively, $T_{\mathrm{f}}$ is the thickness of the thin film, $h$ is the indentation depth at the maximum load, $\alpha_{1}$ is a constant that is determined in the fitting procedure. Menčík et al. ${ }^{[17]}$ proposed also an exponential equation of the similar type as Eq.(5):

$$
\left|\frac{E_{\mathrm{f} / \mathrm{s}}^{*}-E_{\mathrm{s}}^{*}}{E_{\mathrm{f}}^{*}-E_{\mathrm{s}}^{*}}\right|=\mathrm{e}^{\left(-\alpha_{2} \frac{h}{T_{\mathrm{f}}}\right)}
$$

For the flat cylindrical indentation test, assuming the layer materials are purely elastic, Gao et al. ${ }^{[18]}$ also proposed an analytical weight function with the form:

$$
\frac{\left(E^{*}-E_{\mathrm{s}}^{*}\right)}{\left(E_{\mathrm{f}}^{*}-E_{\mathrm{s}}^{*}\right)}=\Phi
$$

where $\Phi$ is the Gao's weight function, given by:

$$
\Phi=\frac{2}{\pi} \arctan \left(\frac{R}{T_{\mathrm{f}}}\right)+\frac{1}{2 \pi(1-\nu)}\left[(1-2 \nu) \frac{R}{T_{\mathrm{f}}} \ln \left(1+\left(\frac{R}{T_{\mathrm{f}}}\right)^{2}\right)-\frac{\frac{R}{T_{\mathrm{f}}}}{1+\left(\frac{R}{T_{\mathrm{f}}}\right)^{2}}\right]
$$

where $\nu$ is the Poisson's ratio and $R$ is the contact radius of a cylindrical flat punch indentation at the maximum load.

In the present study, a new weight expression was proposed to quantify contributions of the film and the substrate to the mechanical behaviors of the film/substrate-system, that is:

$$
C_{\mathrm{fe}} \stackrel{\text { def }}{=}\left|\frac{M_{\mathrm{f} / \mathrm{s}}^{*}-M_{\mathrm{f}}^{*}}{M_{\mathrm{s}}^{*}-M_{\mathrm{f} / \mathrm{s}}^{*}}\right|
$$


where $M_{\mathrm{f}}^{*}, M_{\mathrm{s}}^{*}$ and $M_{\mathrm{f} / \mathrm{s}}^{*}$ are the mechanical parameters of the film, substrate and film/ substrate-system, respectively. For the reduced Young's modulus $E^{*}$, the corresponding weight expression $C_{\mathrm{fe}-E^{*}}$ have some advantages over others since it exhibits a excellent power law dependence on the ratio of $R / T_{\mathrm{f}}$ ( $R$ : radius of the punch, $T_{\mathrm{f}}$ : thickness of the film).

\section{FE Model}

Four different models were concerned in the present study. A solid pressed by rigid indenters can be simplified into a two-dimensional axial symmetric model. The present study concerns two material systems. One is half infinite single-phase material system, the other is film/substrate-system. For each material system, rigid punches with different geometrical sizes were implemented (Figs.2a and 2b). A uniform pressure $p$ (loading history was shown in Fig.1a) with the maximum load $p_{\max }=200 \mathrm{MPa}$ was applied on the punch.

(a)
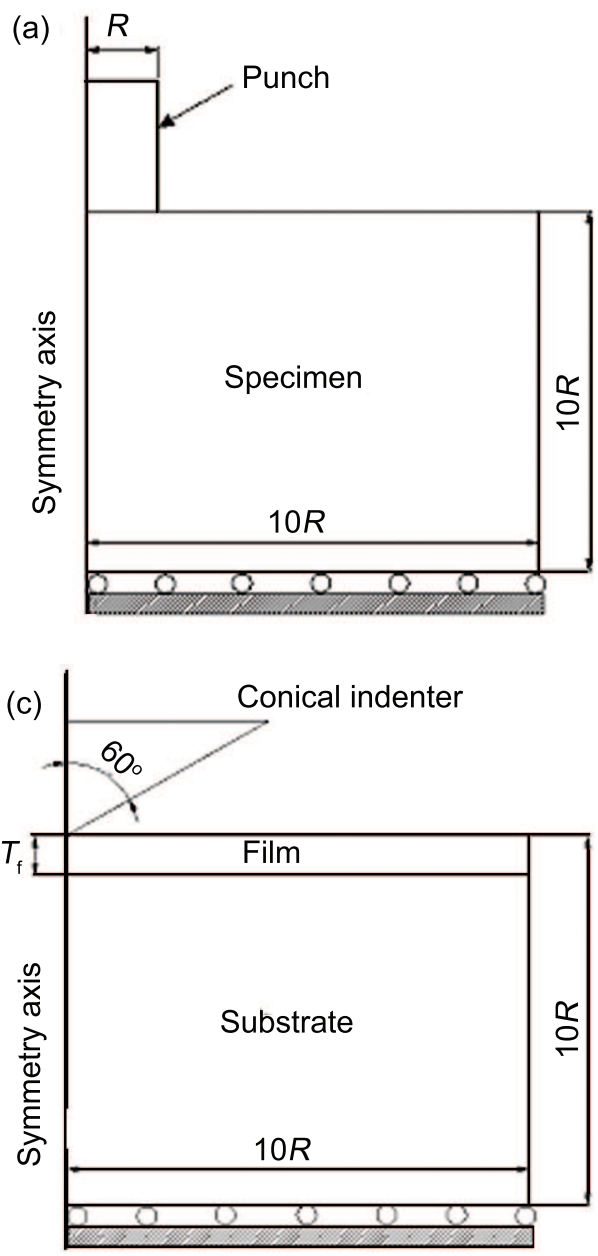

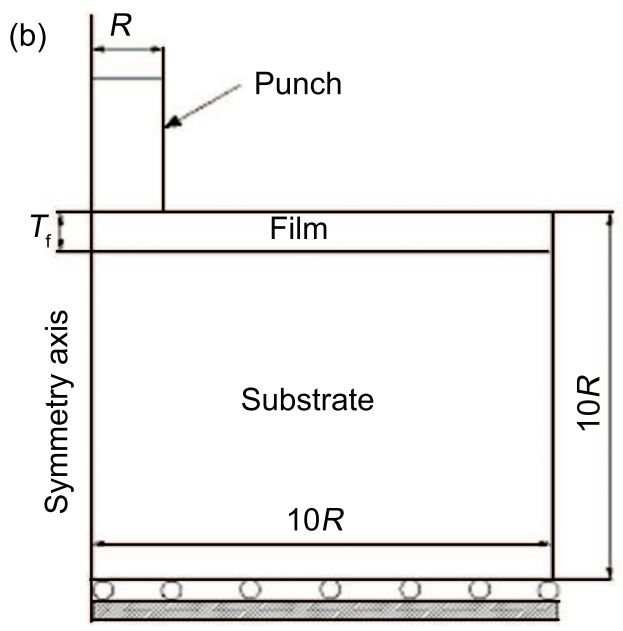

(d)

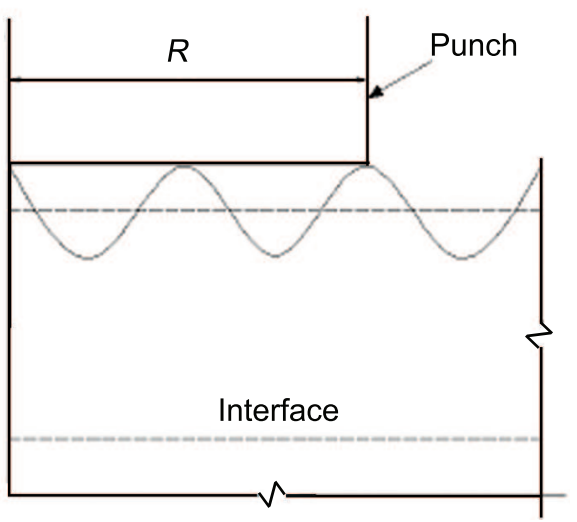

Fig.2 Models used in the present study: (a) half infinite single-phase Material pressed by a punch; (b) film/substrate-system pressed by a punch; (c) film/substrate-system penetrated by a conical indenter; (d) film/substrate-system pressed by a punch incorporating surface roughness. 
A conical indenter with an apex angle of $120^{\circ}$ (Fig.2c) was employed to investigate the maximum depth effect and was compared with the case using a flat-ended punch. The cosine surface profile was incorporated to exploring the effect of surface micro-roughness of thin film on determination of reduced Young's modulus of the film/substrate system (Fig.2d). The surface roughness was quantified by

$$
R_{\mathrm{a}}=\frac{1}{l} \int_{0}^{l}|y(x)| \mathrm{d} x
$$

where $y(x)$ is the height of the surface profile based on centerline, $l$ is the sample length. The mesh design for the rough surface was shown in Fig.3. The mesh at peaks and valleys was refined due to the stress concentration at these places. The size of the tested specimen was chosen $10 R \times 10 R$ so as to ensure the boundary stresses and displacements are insignificant. The thickness of the film $T_{\mathrm{f}}$ was chosen $50 \mu \mathrm{m}$ through out this study. A perfect bonding was assumed to exits between the film and the substrate. Each model was subdivided into about 7,000 two-dimensional meshes with eight-node biquadratic axisymmetric quadrilateral reduced integration elements (CAX8R). Prior to the calculations, our system was optimized in terms of numbers and types of elements to achieve the best compromise between a high accuracy of the calculations and a reasonable computational effort.

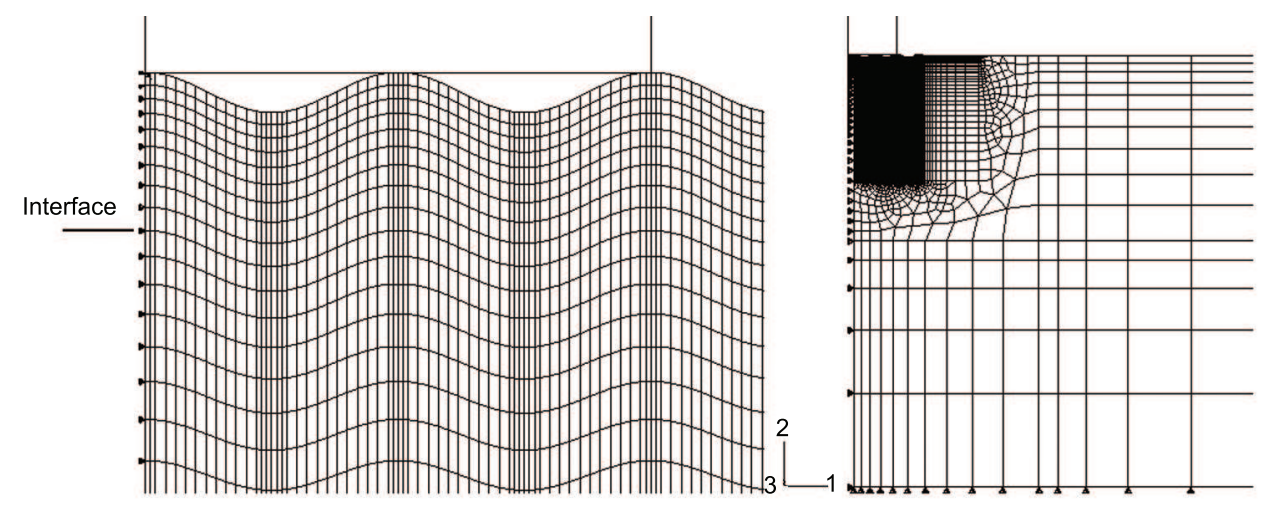

Fig.3 Mesh design for the cosine profile rough surface.

\section{FE Results Analysis}

\subsection{Effect of punch size on distribution of stresses and strain}

Keeping the thickness of the film fixed, we now investigate the effect of punch size on the distribution of von Mises stress at maximum load. The elasto-plastic properties of the film/substrate-system used in this section are presented in Table $1^{[19]}$. Figs.4a and $4 \mathrm{~b}$ shows the distribution of von Mises stress for $R / T_{\mathrm{f}}=0.5$ (Fig. $4 \mathrm{a}$ ) and $R / T_{\mathrm{f}}=5$ (Fig. $4 \mathrm{~b}$ ). Fig.4a clearly shows that stress concentrations occurred in the front of the punch and the interface between the film and substrate. From the stress distribution in Fig.4a, it can be seen that the film is the main supporting layer. When the value of $R / T_{\mathrm{f}}$ increases to 5 (Fig.4b), however, a stress migration can be observed. The maximum stress distributes 
Table 1 Elasto-plastic properties of the film/substrate-system

\begin{tabular}{|c|c|c|c|c|}
\hline & \multicolumn{2}{|c|}{ Film } & \multicolumn{2}{|c|}{ Substrate } \\
\hline Young's modulus/GPa & \multicolumn{2}{|c|}{48} & \multicolumn{2}{|c|}{184} \\
\hline Poisson's ratio & \multicolumn{2}{|c|}{0.1} & \multicolumn{2}{|c|}{0.3} \\
\hline \multirow{7}{*}{ Plastic data } & True stress/MPa & Plastic strain & True stress/MPa & Plastic strain \\
\hline & 50 & 0 & 200 & 0 \\
\hline & 60 & 0.006136 & 240 & 0.0235 \\
\hline & 70 & 0.01238 & 280 & 0.0474 \\
\hline & 85 & 0.02411 & 340 & 0.0935 \\
\hline & 95 & 0.03595 & 380 & 0.1377 \\
\hline & 100 & 0.047 & 400 & 0.18 \\
\hline
\end{tabular}
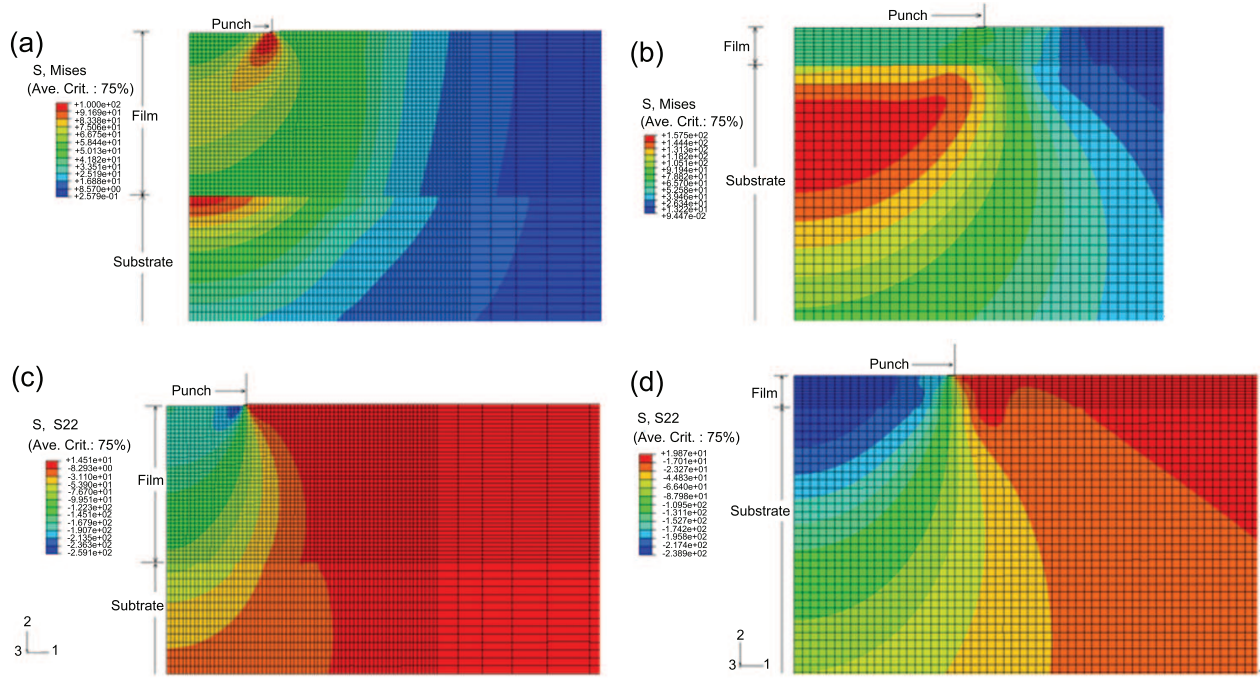

Fig.4 Distribution of stress and strain for different punch sizes: (a) Von Mises stress for $R / T_{\mathrm{f}}=0.5$; (b) Von Mises stress for $R / T_{\mathrm{f}}=5$; (c) strain in 22-direction for $R / T_{\mathrm{f}}=0.5$; (d) strain in 22direction for $R / T_{\mathrm{f}}=5$.

in the substrate and is much larger than that in the film, and the substrate becomes the main supporting layer.

Figs.4c and $4 \mathrm{~d}$ shows the distribution of the strain in 22-direction (refer to the coordinate system) for the two cases of $R / T_{\mathrm{f}}=0.5$ (Fig.4c) and $R / T_{\mathrm{f}}=5$ (Fig. $4 \mathrm{~d}$ ). It can be seen that when the value of $R / T_{\mathrm{f}}=0.5$, the strain in 22-direction mainly distributes in the film layer, while it largely expands to the substrate when the value of $R / T_{\mathrm{f}}$ increases to 5 . For the case $R / T_{\mathrm{f}}=0.5$, the film contributes more to the overall indentation depth than the substrate, while an opposite result can be observed for the case $R / T_{\mathrm{f}}=5$ (the substrate contributes more to the overall indentation depth than the film).

\subsection{Effect of punch size on $C_{\mathrm{fe}-E^{*}}$}

In this section, our emphases were placed on exploring the relationship between $C_{\mathrm{fe}-E^{*}}$ and the value of $R / T_{\mathrm{f}}$. The film thickness $T_{\mathrm{f}}$ was fixed at $0.05 \mathrm{~mm}$, we change the punch size to produce a series of different $R / T_{\mathrm{f}}$ counterparts ranging from 0 to 100 . For each case, the mechanical properties of the film and the substrate are respectively assigned to the whole tested specimen (single-phase material system shown in Fig.2a). Then the film 
and the substrate properties are simultaneously assigned to the film layer and substrate layer, respectively (film/substratesystem shown in Fig.2b). Performing FE calculations for each material system, we obtain the corresponding loading-unloading curves $(F-h)$. The values of $E_{\mathrm{f}}^{*}, E_{\mathrm{s}}^{*}$ and $E_{\mathrm{f} / \mathrm{s}}^{*}$ can be extracted by Eq.(4).

Fig. 5 shows the loading-unloading curves $(F-h)$ resulting from film/substrate-system for different punch sizes. It can be seen that both of indentation depth $h$ and indentation stiffness $S$ increase with the increase of punch size. From Eq.(4) we know that the indentation stiffness $S$ has a linear dependence on the punch radius $R$ for single-phase materials. Fig. 6 gives plots of the reduced Young's modulus of the thin film $E_{\mathrm{f}}^{*}$, the substrate $E_{\mathrm{s}}^{*}$ and the film/substrate-system $E_{\mathrm{f} / \mathrm{s}}^{*}$ as a function of $R / T_{\mathrm{f}}$ for the two cases of $E_{\mathrm{f}}^{*}<E_{\mathrm{s}}^{*}$ and $E_{\mathrm{f}}^{*}>E_{\mathrm{s}}^{*}$. The data is fitted using multipleorder exponential decay curve with a dependency higher than $98 \%$. It is seen that for the single-phase material the reduced Young's modulus is independent on punch size, while for the film/substrate-system the reduced Young's modulus increases with the increase of $R / T_{\mathrm{f}}\left(E_{\mathrm{f}}^{*}<E_{\mathrm{s}}^{*}\right)$. The reduced Young's modulus of the film/substrate-system $E_{\mathrm{f} / \mathrm{s}}^{*}$ approaches to $E_{\mathrm{f}}^{*}$ when the value of $R / T_{\mathrm{f}}$ is small, while it approaches to $E_{\mathrm{s}}^{*}$ when $R / T_{\mathrm{f}} \rightarrow \infty$. Moreover, the value of $E_{\mathrm{f} / \mathrm{s}}^{*}$ is found within intervals which are limited by $E_{\mathrm{f}}^{*}$ (lower limit) and $E_{\mathrm{s}}^{*}$ (upper limit). The corresponding value of $C_{\mathrm{fe}-E^{*}}$ can be obtained by Eq.(9). Fig.7a gives a plot of $C_{\mathrm{fe}-E^{*}}$ as a function of $R / T_{\mathrm{f}}$ for different combinations of $E_{\mathrm{f}}$ and $E_{\mathrm{s}}$. It is seen that $C_{\mathrm{fe}-E^{*}}$ exhibits a good power law dependence on the value of $R / T_{\mathrm{f}}$ with the boundary condition: $R / T_{\mathrm{f}} \rightarrow 0 \Longrightarrow E_{\mathrm{f} / \mathrm{s}}^{*} \rightarrow E_{\mathrm{f}}^{*} \Longrightarrow C_{\mathrm{fe}-E^{*}} \rightarrow 0$. Thus the weight function can be expressed as

$$
C_{\mathrm{fe}-E^{*}}=\left|\frac{E_{\mathrm{f}}^{*}-E_{\mathrm{f} / \mathrm{s}}^{*}}{E_{\mathrm{f} / \mathrm{s}}^{*}-E_{\mathrm{s}}^{*}}\right|=a\left(R / T_{\mathrm{f}}\right)^{b}
$$

This equation was further illustrated in Fig.7b by plotting $C_{\mathrm{fe}-E^{*}}$ in a double logarithmic grid as a function of the corresponding $R / T_{\mathrm{f}}$. A linear dependence of $\lg C_{\mathrm{fe}-E^{*}}$ on $\lg \left(R / T_{\mathrm{f}}\right)$ can be seen in Fig.7b, which is in line with the result of Eq.(11). 

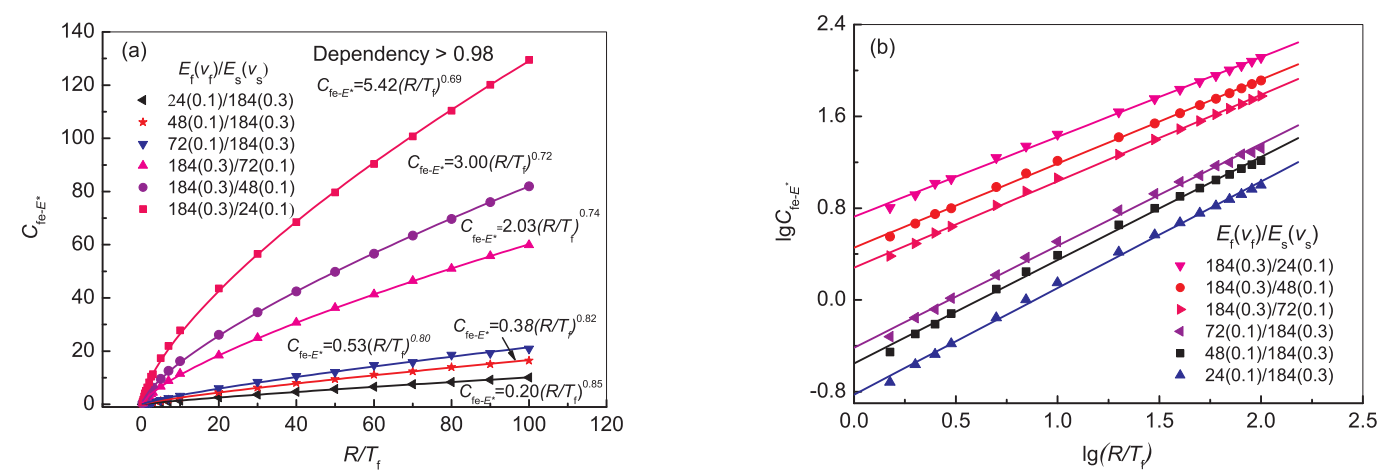

Fig.7 Effect of punch size on $C_{\mathrm{fe}-E^{*}}$. It shows that the data can be well fitted by a power law type function: $C_{\mathrm{fe}-E^{*}}=a\left(R / T_{\mathrm{f}}\right)^{b}$ (the dependency are higher than 0.98). The linear dependence of $\lg C_{\mathrm{fe}-E^{*}}$ on $\lg \left(R / T_{\mathrm{f}}\right)$ shown in (b) is in line with results of (a).

\section{Determination of Film's Reduced Young's Modulus}

\subsection{Weight function method (method 1)}

Generally, the material of the substrate is typical and sufficient, and the elastic constants $E_{\mathrm{s}}$ and $v_{\mathrm{s}}$ can be well calibrated by performing uniaxial tensile testing. In engineering, the reduced Young's modulus of the substrate is generally bigger than that of thin films, from analysis in section 4.2 we have

$$
E_{\mathrm{f}}^{*}<E_{\mathrm{f} / \mathrm{s}}^{*}<E_{\mathrm{s}}^{*}
$$

Assuming the reduced Young's modulus of the substrate $E_{\mathrm{s}}^{*}$ is known, the reduced film's Young's modulus $E_{\mathrm{f}}^{*}$ can be determined by three loading-unloading indentation tests with three different punch sizes $R_{1}, R_{2}$ and $R_{3}$. The three loading-unloading indentation tests are performed on the film/substrate-system, yielding the corresponding reduced Young's modulus $E_{\mathrm{f} / \mathrm{s}-1}^{*}, E_{\mathrm{f} / \mathrm{s}-2}^{*}$ and $E_{\mathrm{f} / \mathrm{s}-3}^{*}$ (Eq.(4)). According to Eq.(11), we have

$$
\lg \left(\frac{E_{\mathrm{f} / \mathrm{s}-1}^{*}-E_{\mathrm{f}}^{*}}{E_{\mathrm{s}}^{*}-E_{\mathrm{f} / \mathrm{s}-1}^{*}} \frac{E_{\mathrm{s}}^{*}-E_{\mathrm{f} / \mathrm{s}-2}^{*}}{E_{\mathrm{f} / \mathrm{s}-2}^{*}-E_{\mathrm{f}}^{*}}\right)=\lg \left(\frac{E_{\mathrm{f} / \mathrm{s}-1}^{*}-E_{\mathrm{f}}^{*}}{E_{\mathrm{s}}^{*}-E_{\mathrm{f} / \mathrm{s}-1}^{*}} \frac{E_{\mathrm{s}}^{*}-E_{\mathrm{f} / \mathrm{s}-3}^{*}}{E_{\mathrm{f} / \mathrm{s}-3}^{*}-E_{\mathrm{f}}^{*}}\right)\left(\frac{\lg R_{1}-\lg R_{2}}{\lg R_{1}-\lg R_{3}}\right)
$$

in which only $E_{\mathrm{f}}^{*}$ is unknown. Eq.(12) can be solved by performing a specially written program. In order to obtain an accurate root of Eq.(12), it is necessary to assess its stability. For simplicity, we define:

$$
m \stackrel{\text { def }}{=} \frac{\lg R_{1}-\lg R_{2}}{\lg R_{1}-\lg R_{3}}
$$

Then Eq.(12) can be written as

$$
\frac{\left(E_{\mathrm{f} / \mathrm{s}-1}^{*}-E_{\mathrm{f}}^{*}\right)^{1-m}\left(E_{\mathrm{f} / \mathrm{s}-3}^{*}-E_{\mathrm{f}}^{*}\right)^{m}}{E_{f / s-2}^{*}-E_{\mathrm{f}}^{*}}=\frac{\left(E_{\mathrm{s}}^{*}-E_{\mathrm{f} / \mathrm{s}-1}^{*}\right)^{1-m}\left(E_{\mathrm{s}}^{*}-E_{\mathrm{f} / \mathrm{s}-3}^{*}\right)^{m}}{E_{s}^{*}-E_{\mathrm{f} / \mathrm{s}-2}^{*}}
$$


We now put our emphasis on the stability of the right side. We define:

$$
f\left(E_{\mathrm{f} / \mathrm{s}-1}^{*}, E_{\mathrm{f} / \mathrm{s}-2}^{*}, E_{\mathrm{f} / \mathrm{s}-3}^{*}\right) \stackrel{\text { def }}{=} \frac{\left(E_{\mathrm{s}}^{*}-E_{\mathrm{f} / \mathrm{s}-1}^{*}\right)^{1-m}\left(E_{\mathrm{s}}^{*}-E_{\mathrm{f} / \mathrm{s}-3}^{*}\right)^{m}}{E_{\mathrm{s}}^{*}-E_{\mathrm{f} / \mathrm{s}-2}^{*}}
$$

The stability condition values with respect to $E_{\mathrm{f} / \mathrm{s}-1}^{*}, E_{f / s-2}^{*}$ and $E_{f / s-3}^{*}$ are:

$$
\begin{aligned}
& \operatorname{Cond} E_{\mathrm{f} / \mathrm{s}-1}^{*}(f)=\left|\frac{E_{\mathrm{f} / \mathrm{s}-1}^{*}}{f} \frac{\partial f}{\partial E_{\mathrm{f} / \mathrm{s}-1}^{*}}\right|=\left|\frac{(1-m) E_{\mathrm{f} / \mathrm{s}-1}^{*}}{E_{\mathrm{s}}^{*}-E_{\mathrm{f} / \mathrm{s}-1}^{*}}\right| \\
& \operatorname{Cond} E_{\mathrm{f} / \mathrm{s}-2}^{*}(f)=\left|\frac{E_{\mathrm{f} / \mathrm{s}-2}^{*}}{f} \frac{\partial f}{\partial E_{\mathrm{f} / \mathrm{s}-2}^{*}}\right|=\left|\frac{1}{\frac{E_{\mathrm{s}}^{*}}{E_{\mathrm{f} / \mathrm{s}-2}} \mid}\right| \\
& \operatorname{Cond} E_{\mathrm{f} / \mathrm{s}-3}^{*}(f)=\left|\frac{E_{\mathrm{f} / \mathrm{s}-3}^{*}}{f} \frac{\partial f}{\partial E_{\mathrm{f} / \mathrm{s}-3}^{*}}\right|=\left|\frac{m E_{\mathrm{f} / \mathrm{s}-3}^{*}}{E_{\mathrm{s}}^{*}-E_{\mathrm{f} / \mathrm{s}-3}^{*}}\right|
\end{aligned}
$$

From what we have learned in section 4.2, we know that the sizes of three punches should be as small as possible to minimize the values of $\operatorname{cond} E_{f / s-1}^{*}(f)$, $\operatorname{cond} E_{f / s-2}^{*}(f)$ and $\operatorname{cond} E_{f / s-3}^{*}(f)$. In other words, a bigger punch radius can induce our method unstable.

\subsection{Numerical procedure (method 2)}

The obvious solution for the correct mechanical property evaluation of thin films requires the use of low loads, in order to obtain only the film's contribution. In order to measure mechanical properties of the film, a commonly used rule is to limit the maximum indentation depth to less than $10 \%$ of the film's thickness ${ }^{[20]}$. However, this procedure cannot be applied to very thin films, for which very low indentation depths are needed. Moreover, it is not easy to manufacture a small indenter (always in nano-scale) due to its high requirement of material hardness and shape accuracy. However, it is possible to predict the film's mechanical parameters by extrapolating the punch radius to zero. Fig. 8 shows how the reduced Young's modulus of the film was obtained using an extrapolation procedure. The details can be realized as follows:

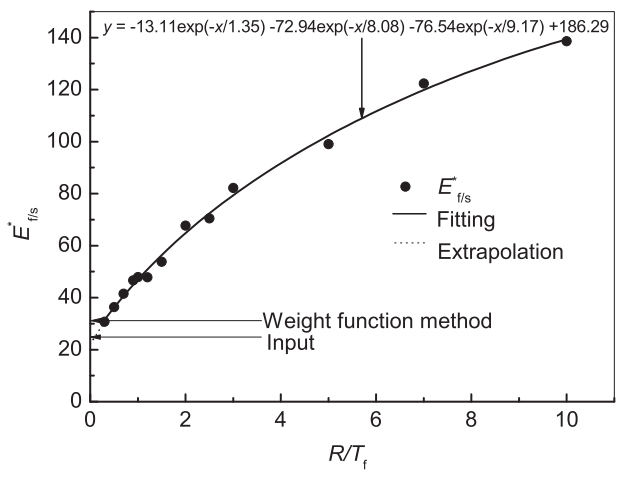

Fig.8 Exponential decay fitting for the extrapolation to film's reduced Young's modulus $(R \rightarrow 0)$.

(1) Performing a series of loading-unloading indentation tests on a film/substrate system with different sizes of punch radius.

(2) Extracting reduced Young's modulus of the film/substrate system corresponding to different punch radius using Eq.(4).

(3) Plotting the reduced Young's modulus of the film/substrate system $E_{\mathrm{f} / \mathrm{s}}^{*}$ as a function of $R / T_{\mathrm{f}}$ ( $R$ : punch radius, $T_{\mathrm{f}}$ : film thickness) and fitting the curve by a multiple-order exponential decay curve. 
(4) Extrapolating the fitting curve to zero punch radius $\left(R / T_{\mathrm{f}} \rightarrow 0\right)$, the corresponding value can be considered as the film's reduced Young's modulus.

The results obtained from the two methods are presented in Table 2. It can be seen that the results obtained by numerical procedure shows a better agreement with the input one. Here it should be pointed out that the weight function method is convenient and suitable for extracting the reduced film's Young's modulus since only three indentation tests with three different punch radius are needed. However, this method is preferred only when the test results are quite reliable, an inaccuracy test will induce a large aberration. More tests need to be performed to get a precise result using least-squares procedure. For the numerical procedure, on the other hand, extensive indentation tests with different punch radius need to be carried out which will be time-assuming and painstaking. However, a more accurate result can be obtained. Method selection should achieve a compromise between test efforts and accuracy of the result.

Table 2 Reduced film's Young's modulus obtained by weight function method and extrapolation method

\begin{tabular}{cccccc}
\hline (Input) $E_{\mathrm{f}}\left(v_{\mathrm{f}}\right) / E_{\mathrm{s}}\left(v_{\mathrm{s}}\right) / \mathrm{GPa}$ & $E_{\mathrm{f}} /\left(1-v_{\mathrm{f}}^{2}\right) / \mathrm{MPa}$ & Method 1/MPa & Error & Method 2/MPa & Error \\
\hline $24(0.1) / 184(0.3)$ & 24242 & 30123 & $24 \%$ & 22409 & $7.6 \%$ \\
$48(0.1) / 184(0.3)$ & 48485 & 59728 & $23 \%$ & 49152 & $1.4 \%$ \\
$72(0.1) / 184(0.3)$ & 72727 & 87617 & $20 \%$ & 85751 & $18 \%$ \\
$184(0.3) / 24(0.1)$ & 202198 & 144934 & $28 \%$ & 160523 & $20 \%$ \\
$184(0.3) / 48(0.1)$ & 202198 & 171172 & $15 \%$ & 188374 & $6.8 \%$ \\
$184(0.3) / 72(0.1)$ & 202198 & 178539 & $12 \%$ & 199918 & $1.1 \%$ \\
\hline
\end{tabular}

\section{Discussion}

\subsection{Effect of maximum indentation depth}

It is reported that the indentation depth strongly influences the reduced Young's modulus of the film/substrate system pressed by a sharp or a ball indenter ${ }^{[16,17]}$. However, the depth effect for a flat-ended punch has rarely reported, and it becomes at least debatable.

Fig.9 shows how the reduced Young's modulus evolves with the increase of maximum indentation depth for a flat-ended punch and a conical sharp indenter. The mechanical properties of the film and substrate are presented in Table 1 . The radius of the punch varies from $0.075 \mathrm{~mm}$ to $1.0 \mathrm{~mm}$.

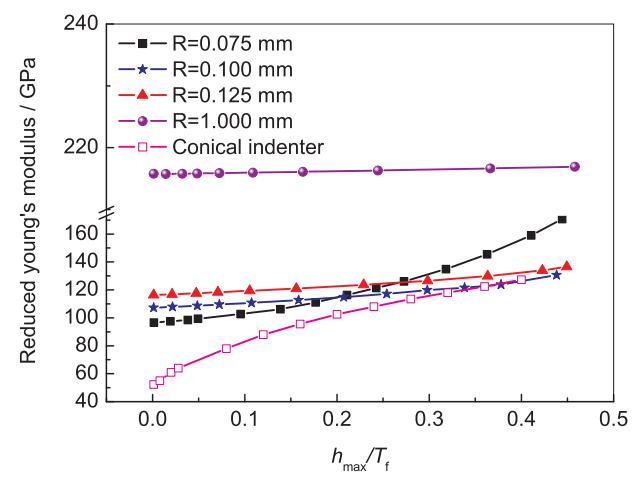

Fig.9 Effect of maximum indentation depth on reduced Young's modulus for a conical indenter and flat punches with different radius.

From Fig.9, three conclusions can be drawn. Firstly, the reduced Young's modulus of the film/substrate system increases monotonously with the increase of the maximum indentation depth for both of the two types of indenters. Secondly, it can be seen that the maximum depth effect associated with a sharp indenter is much more dramatic than that associated with flat-ended punch. The main reason is that the strain gradient under a sharp indenter is more severe. The strain gradient induces the strain energy transmits to the surrounding materials and this can lead to delamination at the interface. Meanwhile, it 
makes the substrate's effect more obvious than that under a flat-ended punch. Thirdly, it can also be seen that a larger punch exhibits a weaker dependence on maximum indentation depth.

It is seen that the substrate's effect can be neglected when the punch radius reaches $1 \mathrm{~mm}$. As applying a uniform pressure $p=200 \mathrm{MPa}$ in the present study, the value of $h_{\max } / T_{\mathrm{f}}$ was limited within intervals of 0 and 0.02 when the punch radius is smaller than $1 \mathrm{~mm}$. Referring to Fig.9, it is acceptable to neglect the effect of maximum indentation depth in the present study.

\subsection{Effect of surface roughness of thin films}

So far we have not considered the possibility that our results are affected by surface roughness of thin films. It is an accepted common rule that the effect of surface roughness can be neglected when the indentation depth is 20 times that of the surface roughness $R_{\mathrm{a}}{ }^{[21]}$. However, how the surface roughness of thin films works on determination of reduced Young's modulus of a film/substrate system is still unclear. In this section, the cosine curve was incorporated to simulate the real rough surface profile. The amplitude of the cosine curve was varied to generate different $R_{\mathrm{a}}$ values, they are $6.4 \mu \mathrm{m}, 12.7 \mu \mathrm{m}$ and $25.5 \mu \mathrm{m}$. The mechanical properties listed in Table 1 were assigned to the film and the substrate. The punch radius was chosen as $R / T_{\mathrm{f}}=3.5\left(T_{\mathrm{f}}=0.05 \mathrm{~mm}\right)$.

Fig.10 shows the load-depth curve for different surface roughness under load $p=100 \mathrm{MPa}$ and $300 \mathrm{MPa}$, respectively. It is seen that the indentation depth increases with the increase of $R_{\mathrm{a}}$ values. Comparing Fig.10a with 10b, we found that this effect was diminished by the increase of load.
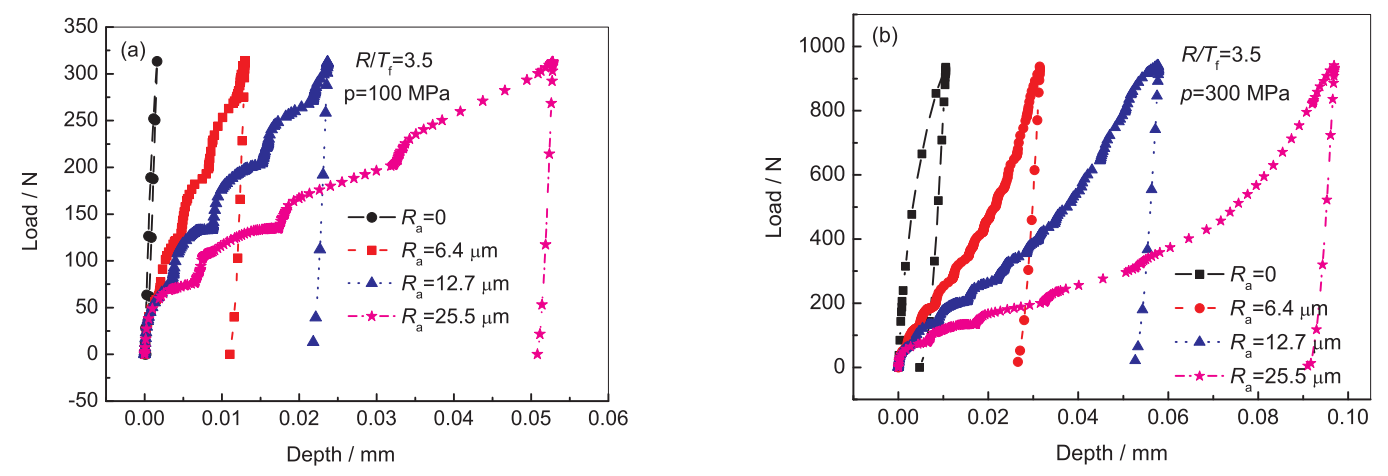

Fig.10 Effect of surface roughness on evolution of load-depth curves: (a) $p_{\max }=100 \mathrm{MPa}$; (b) $p_{\max }=300 \mathrm{MPa}$.

It is interesting to note that the second derivative of the loading curve is negative for a perfect smooth surface $\left(R_{\mathrm{a}}=0\right)$, while it is positive for a rough surface (see Fig.10b). The reason is that the contact area keeps constant for a perfect smooth surface while it keeps increasing for a rough surface during the loading stage until the rough surface is pressed to be flat. This was further illustrated in Fig.11, which shows the deformation maps under load $p=100 \mathrm{MPa}$ and $300 \mathrm{MPa}$ with a surface roughness $R_{\mathrm{a}}=25.5 \mu \mathrm{m}$.

Fig.12 shows the effect of surface roughness on determination of reduced Young's modulus of the film/substrate system. It can be seen that the reduced Young's modulus decreases 
(a)
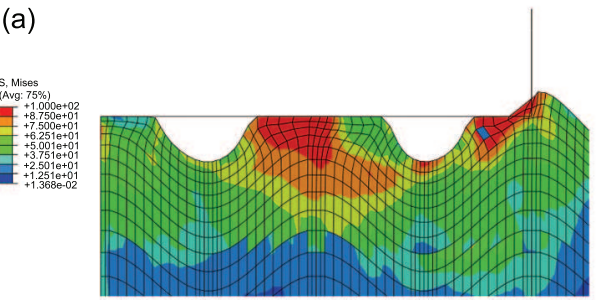

(b)

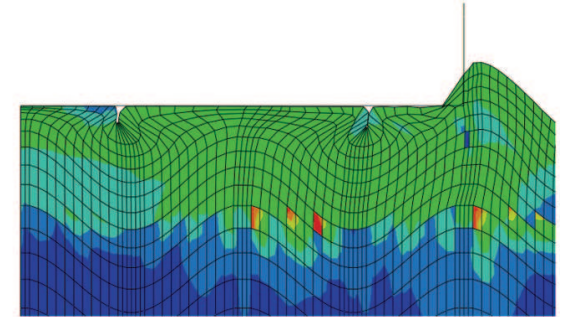

Fig.11 Deformation map of the rough surface: (a) $p=100 \mathrm{MPa}$; (b) $p=300 \mathrm{MPa}$.

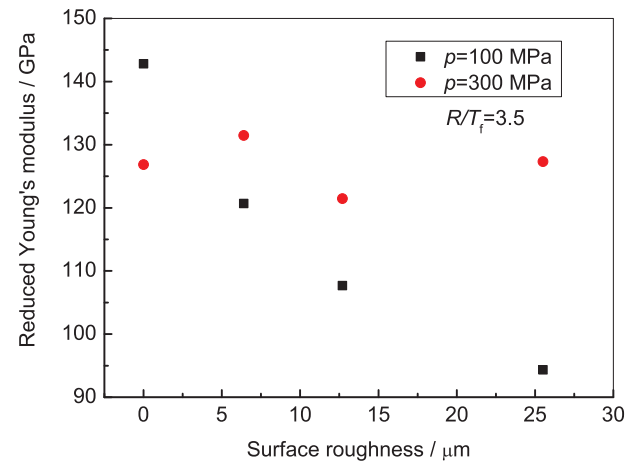

Fig.12 Effect of surface roughness on reduced Young's modulus of the film/substrate system.

with the increase of surface roughness under load $p=100 \mathrm{MPa}$. The reason is that a high value of surface roughness can reduce the effect of substrate effectively. Under a bigger load $p=300 \mathrm{MPa}$, on the other hand, the effect of surface roughness is not that dramatic as aforementioned. This is because the rough surface is pressed to be flat (refer to Fig.11b), which weakened the effect of surface roughness.

\section{Conclusions}

The present study started by an extensive finite element analysis of the loading-

unloading indentation process with different punch radius. Two material systems (singlephase material system and film/substrate-system) were concerned. Our FE calculations were carried out for different classes of materials and punch sizes aimed to develop method to separate elastic properties of a film from a film/substrate-system. FE results showed that when the punch size increased, the stress migrated from film to substrate (the supporting layer transformed from film to substrate), meanwhile the strain largely expanded to the substrate. A new weight function was proposed to relate mechanical properties of the film and substrate with that of film/substrate-system. Based on the weight function, a new method (weight function method) was proposed to separate the reduced film's Young's modulus from a film/substrate-system. At last, a numerical approach including fitting and extrapolating procedures was put forward. It is found that the curve of $E_{\mathrm{f} / \mathrm{s}}^{*} v s R / T_{\mathrm{f}}$ can be well fitted by exponential decay curve, thus the film's reduced Young's modulus can be extracted by extrapolating it to zero punch radius. The maximum depth effect associated with a flat punch is weaker than that associated with a sharp indenter, especially for a large punch. The surface roughness of thin films will reduce substrate's effect under a small load. However, the effect of surface roughness can be diminished by applying a load which is big enough to press the rough surface to be flat. 
- 480 .

\section{REFERENCES}

[1] H.H.K. Xu, D.T. Smith, G.E. Schumacher and F.C. Eichmiller, Dent Mater 16 (2000) 248.

[2] J.M. Antunes, A. Cavaleiro, L.F. Menezes and J.V. Simões, Surf Coat Technol 149 (2002) 27.

[3] D.H. Sastry, Mater Sci Eng A 409 (2005) 67.

[4] G.M. Pharr and W.C. Oliver, Mater Res Bull 17 (1992) 28.

[5] H.J. Kim and Y.G. Kweon, Thin Solid Films 342 (1999) 201.

[6] C.E. Foerster, J.H. Stankievicz and F.C. Serbena, Nucl Instr Meth B 257 (2007) 510.

[7] R.B. King, Int J Solids Struct 23 (1987) 1657.

[8] X. Chen and J.J. Vlassak, J Mater Res 16 (2001) 2974.

[9] J.M. Antunes, L.F. Menezes and J.V. Fernandes, Int J Solids Struct 43 (2006) 784.

[10] Z.F. Yue, G. Eggeler and B. Stöckhert, Comput Mater Sci 21 (2001) 37.

[11] Z.F. Yue, M. Probst-Hein and G. Eggeler, Mater High Temp 17 (2000) 449.

[12] B.X. Xu and Z.F. Yue, J Mater Sci Technol 23(5) (2007) 707.

[13] W.C. Oliver and G.M. Pharr, J Mater Res 7 (1992) 1564.

[14] I.N. Sneddon, Int J Eng Sci 3 (1965) 47.

[15] A.C. Ficher-Cripps, Vacuum 58 (2000) 569.

[16] M.F. Doerner and W.D. Nix, J Mater Res 1 (1986) 601.

[17] J. Menčík, D. Munz and E. Quandt, J Mater Res 12 (1997) 2475.

[18] H.J. Gao, C.H. Chiu and J. Lee, Int J Solids Struct 29 (1992) 2471.

[19] J. Rösler, M. Bäker and K. Aufzug, Acta Mater 52 (2004) 4809.

[20] Y. Sun, T Bell and S. Zheng, Thin Solid Films 258 (1995) 198.

[21] J.Y. Kim, S.K. Kang and J.J. Lee, Acta Mater 55 (2007) 3555. 